\title{
Entrelacs
}

Cinéma et audiovisuel

$7 \mid 2009$

L'atelier

\section{Leçon de cinéma pour notre époque. Politique du sensible}

Lecture

Philippe Ragel

\section{OpenEdition}

\section{Journals}

Édition électronique

URL : http://journals.openedition.org/entrelacs/201

DOI : 10.4000/entrelacs.201

ISSN : 2261-5482

\section{Éditeur}

Éditions Téraèdre

Édition imprimée

Date de publication : 1 mars 2009

Pagination : 187-190

ISBN : 978-2-912868-69-5

ISSN : 1266-7188

\section{Référence électronique}

Philippe Ragel, « Leçon de cinéma pour notre époque. Politique du sensible », Entrelacs [En ligne], 7|

2009, mis en ligne le 01 août 2012, consulté le 21 septembre 2020. URL : http://

journals.openedition.org/entrelacs/201 ; DOI : https://doi.org/10.4000/entrelacs.201

Ce document a été généré automatiquement le 21 septembre 2020.

Tous droits réservés 


\title{
Leçon de cinéma pour notre époque. Politique du sensible
}

\author{
Lecture
}

Philippe Ragel

\section{RÉFÉRENCE}

François Laplantine. Leçon de cinéma pour notre époque Politique du sensible.

Téraèdre / revue Murmure, 2007, 192 p.

1 Après De tous petits liens et surtout Le social et le sensible: introduction à une anthropologie modale, François Laplantine propose avec Leçons de cinéma pour notre époque - Politique du sensible de prolonger son geste d'anthropologue mondialement reconnu, dans le champ du cinéma. Anthropologie et cinéma, drôle d'union diront certains. Mais, rappelle François Laplantine, ce serait là mal connaître d'abord l'histoire du cinéma qui depuis le geste fondateur de Lumière n'a cessé d'observer la réalité ellemême, ensuite oublier combien l'anthropologie et le cinéma sont des «modes de connaissance par l'écoute et le regard " (p. 14) qui obligent une forme d'échange, de partage, partant une éthique. Le mot revient souvent tout au long de cet essai, dessinant une sorte de conduite souterraine que le chercheur applique autant à son endroit qu'au corpus qu'il interroge. Ainsi François Laplantine de prévenir dès les premières pages qu' «il en va de l'éthique et du politique » dans les films dont il traitera, tout en précisant aussitôt « et dans ce livre, d'une démarche qui n'est animée par aucune intention particulière, mais préconise plutôt l'attention» (p. 22). Plus loin, à propos de la ville de Tokyo dans Café Lumière de Hou Hsiao-hsien, l'auteur remarque que sa description «relève ici encore d'une éthique de la connaissance qui, dans le cinéma comme en ethnographie, est une éthique du regard et de l'écoute »(p. 101). En somme, l'anthropologie, et plus précisément le cinéma résume le chercheur, « ça nous regarde dans tous les sens du terme. Ca nous concerne » (p. 22). 
2 Cette posture réflexive qui mobilise autant l'acte de création que de réception, implique des choix dont témoigne le considérable corpus travaillé ici. Dès les premières lignes, en réponse à la question "Que peut faire le cinéma aujourd'hui ? » contre "l'inflation contemporaine des sons et des images qui provoquent une anesthésie des sensations et de la réflexion ", l'auteur nous prévient que cet ouvrage ne traitera pas du «bon gros cinéma... qui drague les spectateurs... les capture en utilisant toutes les ficelles, les trucs et les trucages dont il dispose ", mais "plutôt d'un cinéma délesté des grandes machineries du spectacle, susceptible de revivifier notre rapport à la réalité, de créer de la pensée» (p.13). Nous l'aurons compris, les films élus sous la plume de François Laplantine sont autant des traités de résistance (Résister est d'ailleurs titre du dernier chapitre) « à l'académisme du world cinema qui maquille la réalité, la défigure, dans une pléthore d'images fluorescentes et de sons amplifiés » (p. 29), que des œuvres où notre rapport à la réalité est inquiété, problématique qui constitue l'axe majeur de cette étude. En effet, pour l'auteur «le véritable enjeu de la création cinématographique vient de... la 'tension' entre l'improvisation et la construction » (p. 15), et ne réside surtout pas dans cette manière de cultiver jusqu'à outrance un mode de fabrication qui vise à faire prendre pour du réel ce qui n'en est pas par d' " ingénieux dispositifs de manipulation des émotions» (p. 22). Non plus du reste qu'il résiderait dans la soi-disant objectivité de certains documentaires (la fonction « spéculaire » du cinéma) qu'Agnès Varda avait joliment désignés, rappelle l'auteur, des "documenteurs ". Quand même le corpus considéré privilégie la filiation Lumière à l'héritage Méliès où l'auteur voit, à juste titre, un clivage originel (réalisme vs fantastique), à l'heure des travaux de Pedro Costa ou de Wang Bing auquel l'anthropologue consacre de belles pages, la distinction entre documentaire et fiction ne veut plus dire grand chose : « la séparation entre le documentaire, qui dirait le vrai, et la fiction qui produirait du faux, méconnaît le caractère complexe du rapport aux images au cinéma, qui oscille sans cesse, depuis sa naissance, entre la reproduction fidèle du semblable et l'art du semblant, le miroir et le mirage, bref entre l'influence de Lumière et celle de Méliès « (p. 44). Cette "tension" entre le vrai et le faux, entre le croire et le pas croire qui prolonge d'une certaine manière la thèse deleuzienne de l'interstice, interroge justement la nature même du réel parce qu'elle «introduit du doute...sur le réel lui-même, qui n'a plus alors tout à fait l'évidence dont on le crédite habituellement" (p. 17). Dans ces films qui font retour au réel, qui le mettent à l'épreuve autant que le réel les éprouve, «la dualité du réel et de l 'imaginaire devient problématique» (p. 56); ces films n'ajoutent pas, mais dépouillent nous dit par exemple l'auteur, "renouvelant ainsi ce qu'Ozu a appelé un cinéma à voix basse » ( $\mathrm{p}$. 29). On y travaille les «temps faibles» (chez Raymond Depardon, pp. 105-110) contre les fictions à conflit central qui idolâtre « la dramaturgie (et) frappe de discrédit tout ce qui ne tend pas vers elle » (p. 164); on y découvre « le caractère fantastique de la réalité elle-même" (la pêche aux thons dans Stromboli) contre «les complaisances du naturalisme qui ne visent qu'à convaincre » (p. 58), ou encore l'adiscursivité de certains régimes de narration (de magnifiques pages sur Limite deMario Peixoto auquel l'auteur consacre le chapitre 3 , Troubler) ; on y expérimente enfin la relativité du point de vue chère à l'ethnographe (à propos de Rashômon par exemple). Cette façon de mettre en doute le réel passe aussi par des choix de filmage comme le plan-séquence (Gus VanSant, Angelopoulos, Rouch, Visconti, Welles, Hou Hsiao-hsien, Tarkovski) qui « rapproche les individus» (p. 145) à l'inverse du champ-contrechamp qui divise, se manifeste par des effets de mise en abyme, la mise à distance de la caméra ou son sur- 
rapprochement (24 portraits et Le filmeur d'Alain Cavalier, pp.111-117), ou encore la disparition de la mise en scène au profit d'un dispositif et d'une narration dépourvue des visées téléologiques du modèle dominant (Ten d'Abbas Kiarostami, pp. 119-126).

Du réel, puisqu'il en est beaucoup question à la faveur des films retenus, François Laplantine sait la nature insaisissable: "le réel a une histoire. Il ne cesse de se transformer, il n'est pas identitaire mais procéssuel et événementiel. Bref, il n'a pas la stabilité dont il est le plus souvent crédité » note-t-il page 51. Par leurs hésitations, leurs effets de mise en attente, leurs errances sans but, leurs silences, leurs manques, leurs défauts, comme l'auteur le (dé)montre très bien, toutes ces œuvres en témoignent. Dans leur incomplétude, François Laplantine voit d'ailleurs une forme de prolongement ou d'écho à l'expérience de Wittgenstein et à sa "recherche d'une théorie du sensible (chapitre 2 dans ce livre qui en compte huit) face aux limites ou aux insuffisances du langage supposé impropre à retranscrire, voire à épuiser, une émotion. C'est là peut-être la partie la moins convaincante de ce brillant ouvrage qui n'avait pas besoin de trouver une sorte de caution intellectuelle du côté d'un philosophe dont François Laplantine concède qu'il n'accordait guère d'importance au cinéma. Sur cette question du réel, les travaux de Clément Rosset ou de Jean-Luc Nancy eussent été, nous semble-t-il, plus indiqués. Non tant qu'ils ont tous les deux écrit sur le cinéma mais que, s'agissant du second, il écrivait à propos Kiarostami auquel François Laplantine consacre d'ailleurs de très belles pages, que dans son cinéma " la capture d'image est clairement un ethos, une disposition et une conduite à l'égard du monde » (L'Evidence du film), position qui n'est pas sans rejoindre l'un des nombreux fils rouges qui traversent un ouvrage riche, passionnant à plus d'un titre, militant aussi, et avec lequel, s'agissant de cette question du réel au cinéma, il faudra désormais compter.

\section{AUTEURS}

\section{PHILIPPE RAGEL}

Maître de Conférences à l'Université de Toulouse II le Mirail 